\title{
STANDING CONFERENCE ON UNIVERSITY PROBLEMS (CC-PU) - CONFERENCE REGULIERE SUR LES PROBLEMES UNIVERSITAIRES (CC-PU)
}

\author{
Council of Europe \\ DECS/Higher Education Section \\ B.P. 431 R. 6 \\ 67006 Strasbourg Cedex (France) \\ Tel: (33) 88412622 \\ Fax: (33) 88412788 \\ Telex: eur $870943 \mathrm{f}$ \\ Chairman: Dr. Roberto De Antoniis \\ Secretary: James Wimberley
}

A sub-committee of the Council of Europe's Council for Cultural Cooperation (CDCC), the CC-PU brings together government officials and university members and advises the CDCC on its work programme in higher education, academic mobility and tertiary education. The CC-PU's annual meeting, consisting of a business session and a main theme debate, is held each Spring: a forum role meeting is held in the autumn. Recent main and forum role themes have been "East-West Academic Cooperation" and "the Humboldt Principles of the University and Their Significance for Contemporary Mass Higher Education". Planned main and forum role themes include Universities and Democratization", "Mediterranean Cooperation and European Integration: the Challenge to Universities", and a Conference on Access to Higher Education, to be organized jointly with the Council of Europe's Education Committee. CC-PU activities in the field of Equivalence, recognition and academic mobility are carried out within the NEICs framework (Network of National Equivalence Information Centres).

Principal publications: Council of Europe Student Handbook; Vademecum to University Reception; Structure of University Staff; European Conventions and Agreements on Higher Education (Nos. 15, 21, 32, 69, 138); Recommendations on various issues relating to HigherEducation; Occasional Reports; Annual Programmsof CC-PU Activities. Most publications bilingual (English/French).

La Conférence régulière sur les problèmes universitaires (CC-PU) est un sous-comité du Conseil de la coopération culturelle du Conseil de l'Europe(CDCC). La CC-PU regroupe des hauts fonctionnaires gouvernementaux et des membres des universités et conseille le CDCC sur son programme d'activité dans les domaines de l'enseignement supérieur, de la mobilité académique et de l'enseignement tertiaire. La réunion annuelle du CC-PU, qui comprend une session de travail et un débat sur un thème principal, se tient tous les ans au printemps; un forum a lieu à l'automne. Les derniers thèmes débattus ont porté sur "la coopération universitaire Orient-Occident" et "Le sens des principes Humboldt de l'Université pour l'enseignement supérieur contemporain de masse". Parmi les thèmes de discussion et de forum prévus figurent: "Les universités et la démocratisation”, "La coopération méditerranéenne et l'intégration européenne: un défi aux universités"; de même se tiendra une conférence sur l'accès à l'enseignement supérieur, qui sera organisée conjointement avec le Comité pour l'éducation $d u$ Conseil de l'Europe. Les activités du $C C$-PU dans le domaine des équivalences, de la reconnaissance des diplômes et de la mobilité académique sont menées dans le cadre des Réseaux de centres nationaux d' information sur les équivalences.

Parmi lespublications principales:"Council of Europe Student Handbook"; "Vademecum to University Reception"; "Structure of University Staff"; "European Conventions and Agreements on Higher Education (Nos. 15, 21, $32,69,138)$ "; Recommandations sur certains problèmes relatifs à l' enseignement supérieur; Rapports occasionnels; Programme annuel des activités de la CC-PU. La plupart des publications sont bilingues (français-anglais). 